Research Article

\title{
Space-Time-Frequency Adaptive Processor for Multiple Interference Suppression in GNSS Applications
}

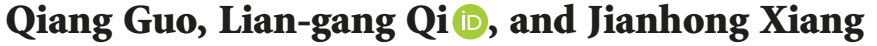 \\ College of Information and Communication Engineering, Harbin Engineering University, Harbin 150001, China \\ Correspondence should be addressed to Lian-gang Qi; qiliangang@hrbeu.edu.cn
}

Received 23 August 2017; Revised 9 January 2018; Accepted 17 January 2018; Published 24 April 2018

Academic Editor: Sotirios K. Goudos

Copyright (c) 2018 Qiang Guo et al. This is an open access article distributed under the Creative Commons Attribution License, which permits unrestricted use, distribution, and reproduction in any medium, provided the original work is properly cited.

\begin{abstract}
To enhance the multiple interference suppression performance of global navigation satellite system (GNSS) receivers without extra antenna elements, a space-time-frequency adaptive processor (STFAP) is investigated. Firstly, based on the analysis of the autocorrelation function of the multicomponent signal, we propose a common period estimation and data block technique to segment the received signal data into blocks. Secondly, the signal data in each block are short-time Fourier transformed into time-frequency (TF) domain, and the corresponding TF points with similar frequency characteristics are regrouped to structure space-time-frequency (STF) data matrixes. Finally, a space-time-frequency minimum output power- (STF-MOP) based weight calculation method is introduced to suppress multiple interfering signals according to their sparse characteristics in TF and space domains. Simulation results show that the proposed STFAP can effectively combat more wideband periodic frequencymodulated (WBPFM) interferences even some of them arriving from the same direction as GNSS signals without increasing the number of antenna elements.
\end{abstract}

\section{Introduction}

In recent years, with the rapidly increasing complexity of the electromagnetic environment, interference suppression techniques for global navigation satellite system (GNSS) receivers have become an increasingly prominent role [1]. Many researchers have been devoting themselves in the study of interference mitigation to ensure the reliability and continuity of GNSS services, and much progress have been already achieved. According to the required number of antenna elements, existing interference suppression methods can be classified into single-antenna interference suppression techniques and multiple-antenna interference suppression techniques. The single-antenna interference suppression methods, such as frequency-domain filtering [2], adaptive time-domain filtering $[3,4]$, and time-frequency (TF) filtering [5], have the advantages of small volume and low hardware complexity; however, they can only deal with the interferences with sparse characteristics in time and frequency domains (such as narrowband interferences and linear chirp interferences) and are not able to cope with multiple interferences [6] effectively. The space processing based on an antenna array, such as power inversion technology and space-only MPDR (S-MPDR) beamformer, can nullify wideband interferences (WBI) and narrowband interferences (NBI) regardless of their time and frequency characteristics [7]. But the number of interferences coped with by spaceonly-based methods is limited to the number of antenna elements. To deal with this shortcoming, the space-time adaptive processing (STAP) is introduced in GNSS applications and widely studied [8-10]. By combining time and spatial processing, it increases the number of suppressed NBI without extra elements in the array; nevertheless, it still cannot deal with the scenario in which the number of WBI exceeds that of antenna elements. With the rapid development of jamming technology and the increasingly complex electromagnetic environment, how to effectively deal with more interferences with a limited number of antenna elements has aroused the concern of people [11]. Recently, [12] drew the attention on suppressing the multiple interferences according to their direction of arrival (DOA) and power by using an open-loop antijam approach. The key idea is to suppress the strong interferences (interference-to-signal ratio (ISR) $>30 \mathrm{~dB}$ ) by spatial processing and ignore the weak 
interferences. Obviously, it failed when the number of strong interferences exceeds that of antenna elements. In addition, the methods mentioned above are not able to cope with the WBI arriving from the same direction as GNSS signals very well. Reference $[13,14]$ drew the attention on cascaded interference suppression methods based on sparse decomposition and spatial filtering. In the first stage, the interfering signals whose waveform characteristics are known are detected and canceled by utilizing their sparsity in the overcomplete dictionary, and the residual interferences are suppressed by spatial filtering in the second stage. However, they need the prior information of interfering signals dealt with by sparse decomposition.

In view of the above problems, this paper proposes a space-time-frequency adaptive processor (STFAP) by combining TF analysis and spatial processing. More specifically, our main contributions are as follows: firstly, in order to avoid the repeated consumption of the spatial degree of freedom (DoF), we propose a common period estimation and data block technique which can obtain the common period of generalized periodic signals and segment received signal data into blocks; then, the signal data in each block are short-time Fourier transformed (STFT) into TF domain, and the corresponding TF points with similar frequency characteristics are regrouped to structure space-timefrequency (STF) data matrixes. Secondly, to avoid the degradation of interference suppression performance when the number of interferences falling into a TF point exceeds the limitation that an antenna array can cope with, we propose a space-time-frequency minimum output power- (STFMOP) based interference suppression method to modify the conventional weight calculation formula by using a reference TF point.

\section{Signal Model}

The analog signals received by an $N$-element antenna array can be expressed as follows:

$$
\mathbf{x}(t)=\mathbf{a} s(t)+\sum_{i=1}^{I} \mathbf{b}_{i} J_{i}(t)+\mathbf{\eta}(t)
$$

where $\mathbf{x}(t)=\left[\mathbf{x}_{1}(t) \mathbf{x}_{2}(t) \cdots \mathbf{x}_{N}(t)\right]^{T}$ is the array signal, each row corresponding to one antenna, and “ $[\bullet]^{T}$ " represents the transpose; $i=1,2, \ldots, I$ represents the number of interfering signals; $\mathbf{a}$ and $\mathbf{b}_{i}$ denote the steering vector of the GNSS signal and the $i$ th interfering signal, respectively; $s, J_{i}$ and $\boldsymbol{\eta}$ are defined as the GNSS signal, the $i$ th interfering signal, and the receiver thermal noise, respectively.

According to their frequency characteristics, interferences can be divided into NBI (e.g., single-tone (S-T) interfering signals) and WBI (e.g., wideband periodic frequencymodulated (WBPFM) and Gaussian noise (WBGN) interfering signals). The WBPFM interfering signal is one of the most efficient interferences due to its wideband and nonstationary characteristics in the frequency domain, and the WBGN interference is considered to be the most costly interference because of the requirement of large transmit power
$[15,16]$. Reference [17] pointed out that using different combinations of multiple WBPFM and S-T interfering signals and fewer WBGN interferences, we can effectively disable the STAP with lower costs. Therefore, this paper focuses on the research of mixed interference suppression in the presence of these interfering signals. In general, the S-T and WBPFM interfering signals can be expressed as follows:

$$
J_{M_{k}}(t)=A_{k} e^{-j 2 \pi\left(f_{M_{k}}(t)+f_{k}+\varphi_{k}\right)},
$$

where $f_{M_{k}}(\bullet)$ is the frequency-modulated (FM) function with the period $T_{k}$, where $k=1,2, \ldots, K$ represents the number of WBPFM interfering signals; $A_{k}, f_{k}$, and $\varphi_{k}$ are the amplitude, the carrier frequency, and the phase, respectively.

\section{The Proposed STFAP}

From [1], we have known that, in a relatively short time, the bandwidth of the WBPFM signal is narrow; however, since its frequency is rapidly varying compared to the receiver integration time, it acts like a WBI. And as we have known, the time domain finite impulse response (FIR) filter in the conventional STAP is not able to make full use of the timefrequency sparse characteristics of interference signals because it only has frequency-resolving ability. Then, the WBPFM interference is treated as a global WBI. It results in the waste of the spatial DoF.

It is aware that since WBPFM interfering signals have significant concentrated energy distribution and periodicity in the time-frequency domain, there are a few TF points affected by the interferences. For the convenience of expression, an analysis is carried out by taking two linear frequency-modulated continuous wave (LFMCW) interfering signal as an example, and their spectrogram is shown in Figure 1, where $f_{c}$ and $B$ are the carrier frequency and bandwidth, respectively; $f_{m_{\mathrm{f}}}, m_{\mathrm{f}}=1,2, \ldots, M_{2}$, denotes the frequency bins; $T_{c}$ and $l$ represent the integer multiple of two modulation period of the two LFMCW interfering signals and a positive integer, respectively; and $t_{l, m_{\mathrm{t}}} m_{\mathrm{t}}=$ $1,2, \ldots, M_{1}\left(m_{\mathrm{t}}=1,2, \cdots, M_{1}\right)$ is the number of signal data in time domain. And the TF point $\left(t_{l, m_{\mathrm{t}}}, f_{m_{\mathrm{f}}}\right)$ is named as the $\left(m_{\mathrm{t}}, m_{\mathrm{f}}\right)$ th TF point belonging to the $l$ th block, and we defined that the " $\left(m_{\mathrm{t}}, m_{\mathrm{f}}\right)$ th TF point" represents the " $\left(m_{\mathrm{t}}, m_{\mathrm{f}}\right)$ th TF point" belonging to all blocks. From it, we can find out that although the bandwidth of the two interfering signals are the same, their frequencies are different at most times due to their different modulation periods and initial frequencies. In theory, WBPFM interferences can be treated as NBI by using a single TF point data, and the number of interferences falling into one TF point may be less than the total number of interferences in the receiving environment. However, we need huge amounts of snapshot data to ensure the performance of interference suppression due to the unknown nature of interferences and the thermal noise. Looking closely at Figure 1, the frequency characteristics of the two interfering signals in TF points $\left(t_{l, m_{\mathrm{t}}}, f_{m_{\mathrm{f}}}\right)$ and $\left(t_{l+l_{\mathrm{n}}, m_{\mathrm{t}}}, f_{m_{\mathrm{f}}}\right)$, such as $\left(t_{1,1}, f_{1}\right)$ and $\left(t_{2,1}, f_{1}\right)$, are consistent, where $l_{\mathrm{n}}$ is a positive integer. In other words, all of the 


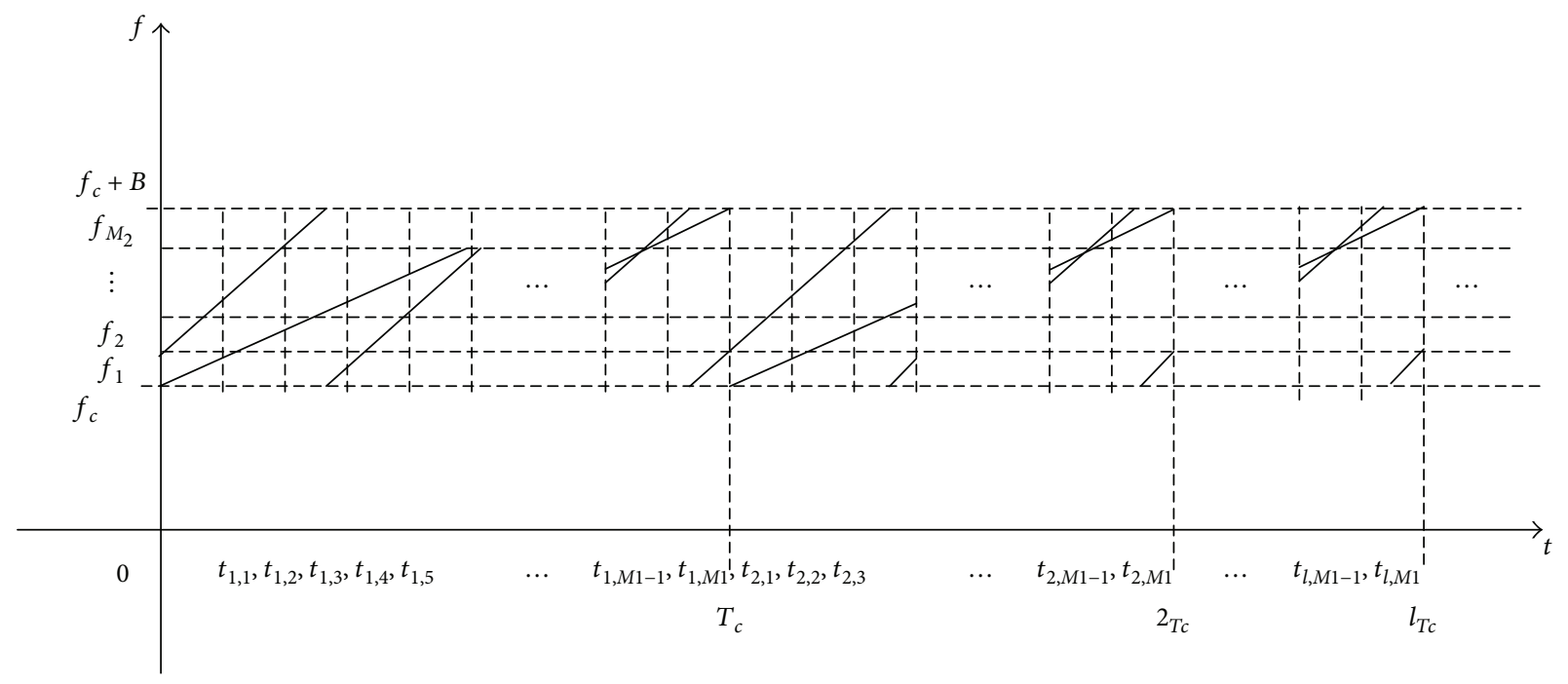

FIGURE 1: The frequency variation of two LFMCW interfering signals.

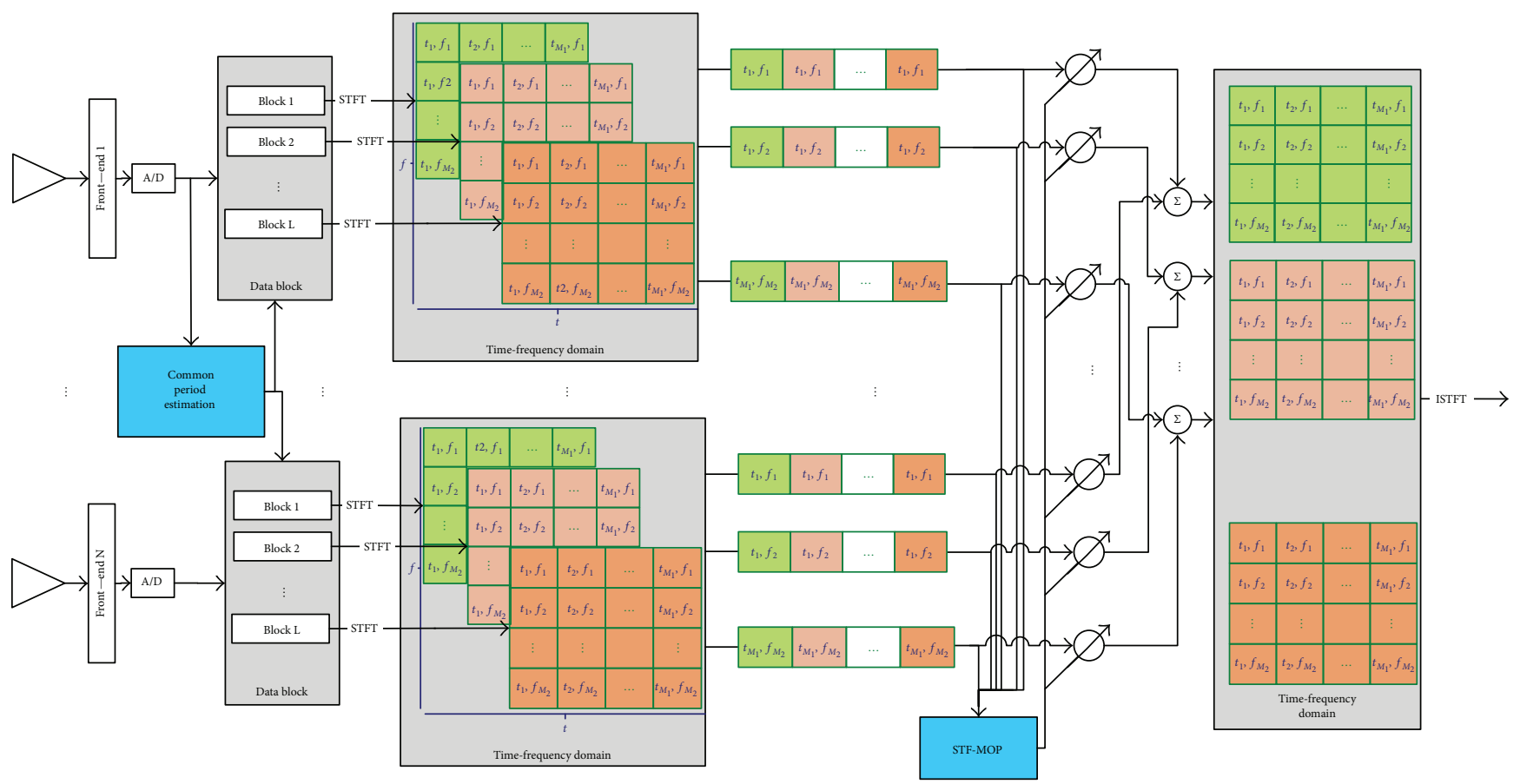

FIgURE 2: The diagram of the proposed STFAP.

$\left(m_{t}, m_{f}\right)$ th TF points consist the same WBPFM interferences. Then, we are able to regroup the TF points with similar TF characteristics to obtain the required number of snapshot data.

Accordingly, we proposed a STFAP which is to improve the multiple interference suppression performance of GNSS receivers by using sparse characteristics of interfering signals in time, frequency, and space domains. The diagram of the proposed STFAP is shown in Figure 2. It can be found that compared with the conventional multiple-antenna interference suppression techniques, such as S-MPDR beamformer and STAP, the proposed method requires no extra antenna elements, RF modules, and analog to digital conversion modules. The difference between conventional methods and the proposed one is the digital signal processing. And the proposed method is composed of the common period estimation and data block technique, the STF data matrix construction method, and the STF-MOP-based interference suppression. Firstly, the unbiased autocorrelation analysis is carried out for the received signal data in one of the channels to estimate the common period of WBPFM interfering signals. Then, the received signal data in all channels are segmented into blocks according to the estimated period. Secondly, the signal data in each block are STFT into TF domain, and the corresponding TF points with similar frequency characteristics are grouped to structure STF data 
matrixes. Thirdly, the optimum weight vectors are computed by using STF-MOP-based weight calculation method, and the TF data after interference suppressing are inverse shorttime Fourier transformed (ISTFT) into the time domain.

\subsection{The Autocorrelation-Based Common Period Estimation} and Data Block Technique. Since the energy of GNSS signals at ground receivers is much lower than that of the receiver thermal noise, they are able to be negligible in the following analysis. Then, the signal in the $n$th channel can be written as follows:

$$
\mathbf{x}_{n}(t)=\sum_{k=1}^{K} J_{M_{k}}(t)+\aleph(t)
$$

where $J_{M_{k}}$ represents the $k$ th WBPMF interfering signal; $\aleph(t)=\sum_{i=1}^{I-K} J_{g_{i}}(t)+\eta_{n}(t)$, in which $J_{g_{i}}$ and $\eta_{n}$ are the $i$ th WBGN interfering signal and the receiver thermal noise corresponding to the $n$th channel, respectively. Since $J_{g_{i}}$ and $\eta_{n}$ are independent of each other, $\aleph$ can be regarded as a WBGN. Because all signals are independent of each other, the cross-correlation function of different signals is 0: the autocorrelation function of $\mathbf{x}_{n}$ should be as follows:

$$
R_{x}(\tau)=\sum_{i=1}^{K} R_{M_{k} M_{k}}(\tau)+R_{\aleph \aleph}(\tau),
$$

where $R_{M_{k} M_{k}}$ and $R_{\aleph N}$ are the autocorrelation function of the $k$ th WBPFM interfering signal and the autocorrelation function of $\aleph$, respectively. Since $\aleph$ is the Gaussian white noise, $R_{\aleph \mathcal{N}}(\tau)=0(\tau \neq 0)$. And the autocorrelation function of the periodic signal is still a periodic function. Then, when $\tau \neq 0$,

$$
\begin{aligned}
\left|R_{x}(\tau)\right| & =\left|\sum_{k=1}^{K} R_{M_{k} M_{k}}(\tau)\right| \\
& =\left|\sum_{k=1}^{K} \frac{A_{k}^{2}}{2} e^{-j 2 \pi f_{k} \tau} \lim _{T \rightarrow \infty} \frac{1}{T} \int_{T}^{-T} e^{-j 2 \pi\left(f_{M_{k}}(t)-f_{M_{k}}^{*}(t-\tau)\right)} d t\right| \\
& \leq \sum_{k=1}^{K} \frac{A_{k}^{2}}{2}
\end{aligned}
$$

where " $(\bullet)^{* \prime}$ represents the conjugate. If and only if $f_{M_{k}}(t)$ $-f_{M_{k}}(t-\tau)=0$ and $\left(f_{k_{i}}-f_{k_{j}}\right) \tau=\alpha$, in which $\alpha$ is an integer, the equality holds. Because $f_{M_{k}}$ is a periodic function over $\tau$ and the period is $T_{k},\left|R_{x}(\tau)\right|$ has many maximums which appear at $T_{\mathrm{c}}$.

$$
\left\{T_{\mathrm{c}} \mid T_{\mathrm{c}}=\left[T_{1}, T_{2}, \ldots, T_{K}\right]_{C M} \&\left(f_{k_{i}}-f_{k_{j}}\right) T_{\mathrm{c}}=\alpha\right\},
$$

where " $[\bullet]_{C M}$ " is defined as the function for computing the common multiple of a list of numbers.

Based on the above analysis, an autocorrelation-based common period estimation method in practical applications is proposed. And the details are described as follows. The digital signals with sampling period $T_{\mathrm{s}}$ can be written as follows:

$$
x(m)=\left[x_{1}(m) x_{2}(m) \cdots x_{N}(m)\right]^{T} .
$$

And the unbiased estimation of the autocorrelation function can be obtained by using finite-length received signal data.

$$
\left|\widehat{R}_{x}\left(m_{\tau}\right)\right|=\left|\frac{1}{M_{\mathrm{R}}-m_{\tau}} \sum_{m=m_{\tau}+1}^{M_{\mathrm{R}}} x_{n}(m) x_{n}^{*}\left(m-m_{\tau}\right)\right|,
$$

where $M_{\mathrm{R}}$ is the length of sampled signal data. Because it is impossible to get infinite-length sampled signal data, the autocorrelation function of WBGN may not be 0 when $m_{\tau} \neq 0$. And the maximums of $\left|\widehat{R}_{x}\left(m_{\tau}\right)\right|$ appearing at $T_{c}$ may not have strict equality. To estimate the common period of multiple WBPFM interfering signals, eliminate the value of $\left|\widehat{R}_{x}\left(m_{\tau}\right)\right|$ near $m_{\tau}=0$, then a normalization processing is carried out:

$$
\left|\widehat{R}_{c}\left(m_{\tau}\right)\right|=\left\{\begin{array}{l}
0,0 \leq m_{\tau} \leq m_{0}, \\
\frac{\left|\widehat{R}_{x}\left(m_{\tau}\right)\right|}{\max \left(\left|\widehat{R}_{x}\left(m_{\tau}\right)\right|\right)}, \quad \text { others }
\end{array}\right.
$$

where $m_{0}$ is the minimum of the search range of the common period. Then, the time corresponding to the first peak which is greater than $\rho$ from $m_{\tau}=0$ is the block size $M_{\mathrm{c}}\left(\widehat{T}_{\mathrm{c}}=M_{\mathrm{c}} T_{\mathrm{s}}\right.$ is the estimated common period). $\rho(0.5<\rho<1)$ is a threshold to judge whether the peak is required.

Let the number of blocks in every channel be $L$, then the signal vector of the $l$ th block at the $n$th channel can be written as follows:

$$
X_{n l}=\left[\mathbf{x}_{n}\left((l-1) M_{\mathrm{c}}+1\right), \ldots, \mathbf{x}_{n}\left((l-1) M_{\mathrm{c}}+M_{\mathrm{c}}\right)\right]^{T} .
$$

3.2. The STF Data Matrix Construction Method. The digital signal data in each block are STFT into TF domain:

$$
X_{n}\left(m_{\mathrm{t}}, m_{\mathrm{f}}, l\right)=\sum_{m} X_{n l}(m) \omega\left(m_{\mathrm{t}}-m\right) e^{-j\left(2 \pi / M_{\mathrm{c}}\right) m_{\mathrm{f}} m},
$$

where " $\omega(\bullet)$ " represents the analysis window; $m_{\mathrm{t}}=1,2, \ldots$, $M_{1}$ and $m_{\mathrm{f}}=1,2, \ldots, M_{2}$ denote the number of sampled data and the number of frequency bins, respectively; $X_{n}\left(m_{t}, m_{f}, l\right)$ is named as the " $\left(m_{\mathrm{t}}, m_{\mathrm{f}}\right)$ th TF point" belonging to the $l$ th block in the $n$th channel, which represents the TF characteristics of signals in the corresponding TF point. And WBPFM interferences in the $\left(m_{\mathrm{t}}, m_{\mathrm{f}}\right)$ th TF points belonging to all blocks have similar TF characteristics. To obtain enough snapshot data with similar frequency characteristics, we should regroup the TF point data. Then, every batch of the $\left(m_{\mathrm{t}}, m_{\mathrm{f}}\right)$ th TF points should be treated as an input matrix of spatial filtering:

$$
\mathbf{X}_{\left(m_{\mathrm{t}}, m_{\mathrm{f}}\right)}=\left[X_{1_{\left(m_{\mathrm{t}}, m_{\mathrm{f}}\right)}}, X_{2_{\left(m_{\mathrm{t}}, m_{\mathrm{f}}\right)}}, \ldots, X_{N_{\left(m_{\mathrm{t}}, m_{\mathrm{f}}\right)}}\right]^{T}
$$

in which

$X_{n_{\left(m_{\mathrm{t}}, m_{\mathrm{f}}\right)}}=\left[X_{n}\left(m_{\mathrm{t}}, m_{\mathrm{f}}, 1\right), X_{n}\left(m_{\mathrm{t}}, m_{\mathrm{f}}, 2\right), \ldots, X_{n}\left(m_{\mathrm{t}}, m_{\mathrm{f}}, L\right)\right]^{T}$. 
TABLE 1: Interference signal characteristics.

\begin{tabular}{lcccccc}
\hline Name & Type & DOA & Broadband & CF & $T_{\mathrm{FM}}(\mu \mathrm{s})$ & INR $(\mathrm{dB})$ \\
\hline 1 & S-T & $50^{\circ}$ & 0 & $1.023 \mathrm{MHz}$ & 55 \\
2 & LFMCW & $80^{\circ}$ & $2 \mathrm{MHz}$ & $1.023 \mathrm{MHz}$ & 31.25 & 55 \\
3 & LFMCW & $110^{\circ}$ & $2 \mathrm{MHz}$ & $1.023 \mathrm{MHz}$ & 20.83 \\
4 & $30^{\circ}$ & $2 \mathrm{MHz}$ & $1.023 \mathrm{MHz}$ & 62.56 & 50.05 \\
5 & SFMCW & $165^{\circ}$ & $2 \mathrm{MHz}$ & $1.023 \mathrm{MHz}$ & $/$ & 55 \\
6 & SFMCW & $20^{\circ}$ & $2 \mathrm{MHz}$ & $1.023 \mathrm{MHz}$ & 50 \\
7 & WBGN & $155^{\circ}$ & $2 \mathrm{MHz}$ & $1.0 \mathrm{MHz}$ & 50 \\
\hline
\end{tabular}

3.3. The STF-MOP-Based Weight Calculation Method. The MPDR beamformer is employed to nullify the interferences in each batch of TF points, whose optimization problem can be expressed as follows:

$$
\min _{\mathbf{w}} \mathbf{w}_{\left(m_{\mathrm{t}}, m_{\mathrm{f}}\right)}^{H} \mathbf{R}_{\left(m_{\mathrm{t}}, m_{\mathrm{f}}\right)} \mathbf{w}_{\left(m_{\mathrm{t}}, m_{\mathrm{f}}\right)} \quad \text { s.t. } \quad \mathbf{w}_{\left(m_{\mathrm{t}}, m_{\mathrm{f}}\right)}^{H} \mathbf{a}=1,
$$

where $\mathbf{w}_{\left(m_{\mathrm{t}}, m_{\mathrm{f}}\right)}$ represents the array weight vector for the $\left(m_{\mathrm{t}}, m_{\mathrm{f}}\right)$ th TF point; $\mathbf{R}_{\left(m_{\mathrm{t}}, m_{\mathrm{f}}\right)}$ is the covariance matrix estimated by using the maximum likelihood criterion:

$$
\mathbf{R}_{\left(m_{\mathrm{t}}, m_{\mathrm{f}}\right)}=\frac{1}{L} X_{\left(m_{\mathrm{t}}, m_{\mathrm{f}}\right)}^{H} X_{\left(m_{\mathrm{t}}, m_{\mathrm{f}}\right)},
$$

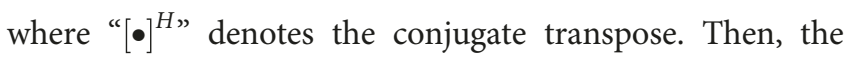
optimal weight vector is

$$
\mathbf{w}_{\left(m_{\mathrm{t}}, m_{\mathrm{f}}\right)_{\mathrm{opt}}}=\frac{\mathbf{R}_{\left(m_{\mathrm{t}}, m_{\mathrm{f}}\right)}^{-1} \mathbf{a}}{\mathbf{a}^{H} \mathbf{R}_{\left(m_{\mathrm{t}}, m_{\mathrm{f}}\right)}^{-1} \mathbf{a}}=\beta_{\left(m_{\mathrm{t}}, m_{\mathrm{f}}\right)} \mathbf{R}_{\left(m_{\mathrm{t}}, m_{\mathrm{f}}\right)}^{-1} \mathbf{a} .
$$

As shown in (16), $\mathbf{w}_{\left(m_{\mathrm{t}}, m_{\mathrm{f}}\right)_{\mathrm{opt}}}$ is calculated by only using the information of the $\left(m_{\mathrm{t}}, m_{\mathrm{f}}\right)$ th TF point and having nothing to do with other TF points. And $\beta_{\left(m_{\mathrm{t}}, m_{\mathrm{f}}\right)}$ is real and makes all weights in the same order of magnitude. But when the number of interference falling into a TF point exceeds the limitation that an antenna array can cope with or there are interferences arriving from the same direction as GNSS signals, it will naturally cause the interferences not to be suppressed. In order to solve these problems, a STF-MOPbased weight calculation method by using the information of the TF point whose energy is minimal is proposed, which can be expressed mathematically as follows:

$$
\mathbf{w}_{\left(m_{\mathrm{t}}, m_{\mathrm{f}}\right)_{\text {opt }}}=\frac{\mathbf{R}_{\left(m_{\mathrm{t}}, m_{\mathrm{f}}\right)}^{-1} \mathbf{a}}{C+\mathbf{a}^{H} \mathbf{R}_{\left(m_{\mathrm{t}}, m_{\mathrm{f}}\right)}^{-1}},
$$

where $C$ is defined as follows:

$$
\begin{aligned}
\left(m_{\mathrm{t}}^{\max }, m_{\mathrm{f}}^{\max }\right) & =\arg \max _{\left(m_{\mathrm{t}}, m_{\mathrm{f}}\right)} \mathbf{a}^{H} \mathbf{R}_{\left(m_{\mathrm{t}}, m_{\mathrm{f}}\right)}^{-1} \mathbf{a}, \\
C & =\mathbf{a}^{H} \mathbf{R}_{\left(m_{\mathrm{t}}^{\max }, m_{\mathrm{f}}^{\max }\right)}^{-1} \mathbf{a} .
\end{aligned}
$$

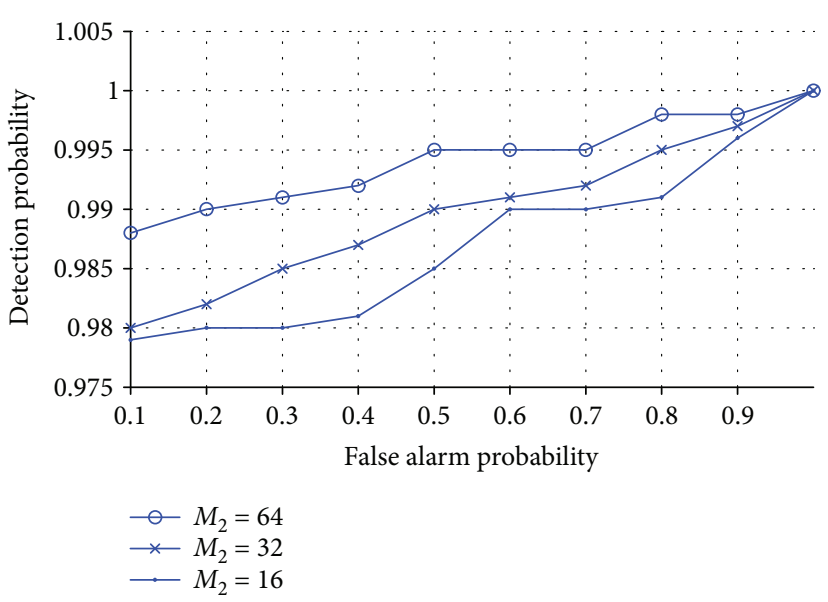

Figure 3: ROC curves of different $M_{2}$.

Then, the TF signal data after spatial filtering are as follows:

$$
Y\left(m_{\mathrm{t}}+(l-1) M_{\mathrm{c}}, m_{\mathrm{f}}\right)=\mathbf{w}_{\left(m_{\mathrm{t}}, m_{\mathrm{f}}\right)_{\mathrm{opt}}}^{H} X_{\left(m_{\mathrm{t}}, m_{\mathrm{f}}\right)}(:, l),
$$

where $X_{\left(m_{\mathrm{t}}, m_{\mathrm{f}}\right)}(:, l)$ is the $l$ th column of matrix $\mathbf{X}_{\left(m_{\mathrm{t}}, m_{\mathrm{f}}\right)}$.

Finally, $Y$ is ISTFT into the time domain to obtain the output data for subsequent processing.

\section{Experiments and Simulation Results}

Two simulations have been carried out to assess the performance of the proposed method. In all simulations, a linear half-wavelength space antenna array with 4 elements is adopted. And there is only one GNSS signal operating on $1.023 \mathrm{MHz}$ with $\mathrm{C} / \mathrm{A}$ code, whose $\mathrm{DOA}$ is $80^{\circ}$. If there is no special explanation, the analog signal with signal-to-noise ratio $(\mathrm{SNR}=-15 \mathrm{~dB})$ is sampled at $4.096 \mathrm{MHz}$. The parameters of interference signals are shown in Table 1 , where $T_{\mathrm{FM}}$ is the period of FM; LFMCW, SFMCW, and CF represent the linear frequency modulation, sinusoid frequency modulation interferences, and the center frequency of WBI, respectively. And the length of $\omega(\bullet)$ is $65 ; L=20 ; \rho=0.95$.

4.1. Influence of $M_{2}$ on the Performance of the Proposed Method. To show the influence of $M_{2}$ on the performance of the proposed method, an interference scenario in the presence of all the interfering signals listed in Table 1 has been 
TABLE 2: Computation complexities of the proposed method and the compared methods.

\begin{tabular}{lcccc}
\hline Name & DCQGMP-SD & Periodic estimation & STFT and ISTFT & Interference suppression \\
\hline S-MPDR & $/$ & $/$ & $/$ & $\mathrm{O}\left(N^{3}\right)$ \\
ST-MPDR & $/$ & $/$ & $/$ & $\mathrm{O}\left(N_{\tau}^{3} N^{3}\right)$ \\
DCQGMP-SD \& MPDR & $\mathrm{O}\left(N_{\mathrm{J}} N_{\mathrm{G}} N_{\mathrm{Q}} N_{\mathrm{S}}^{2}\right)$ & $/$ & $\mathrm{O}\left(N^{3}\right)$ & $\mathrm{O}\left(M_{1} M_{2} N^{3}\right)$ \\
Proposed & $/$ & $\mathrm{O}\left(N_{\mathrm{s}} \log _{2}\left(N_{\mathrm{s}}\right)\right)$ & $\mathrm{O}\left(N_{\omega} M_{2} \log _{2}\left(M_{2}\right)\right)$ & \\
\hline
\end{tabular}

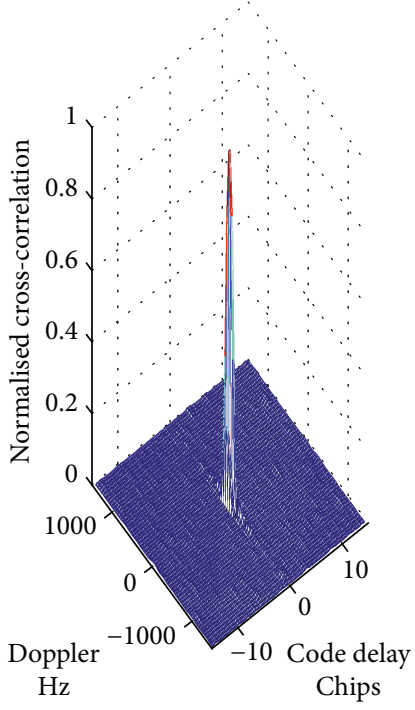

(a) S-MPDR

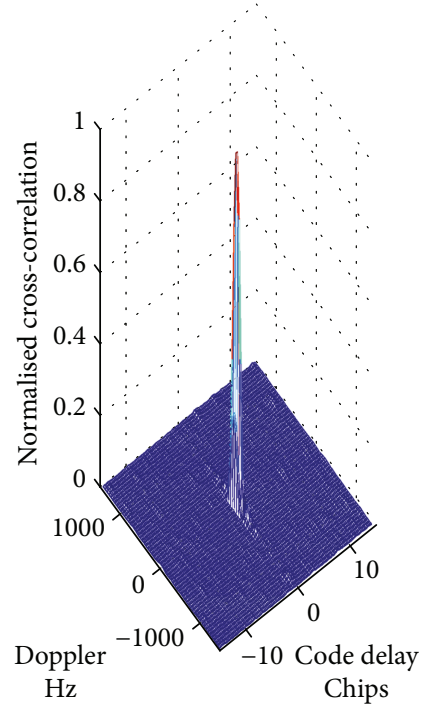

(b) DST-MPDR

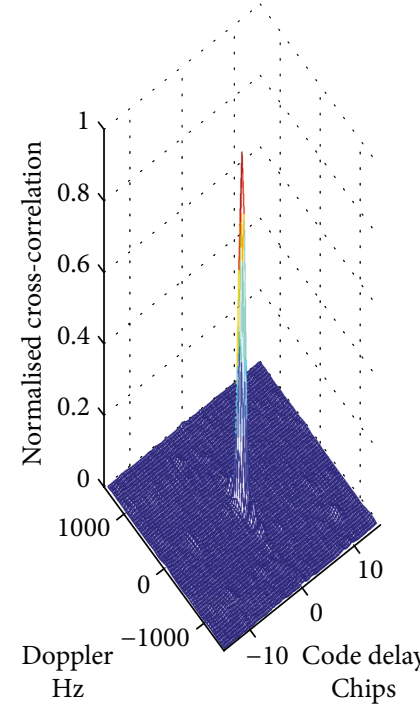

(c) DCQGMP-SD \& MPDR

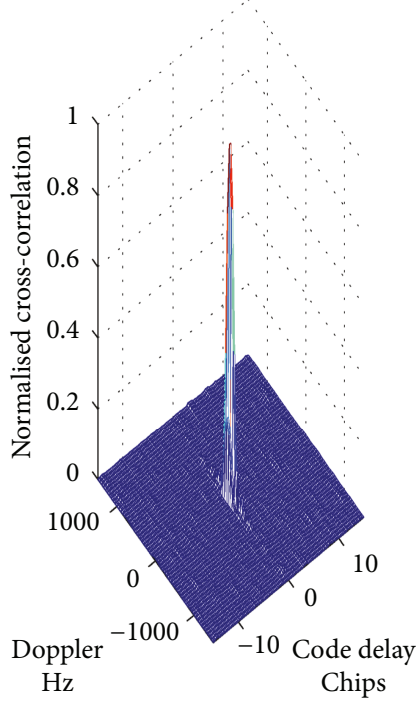

(d) Proposed

FIGURE 4: Correlation peaks after interference suppression for scenario I.

considered. Then, the receiver operating characteristic (ROC) curves obtained from 500 Monte Carlo are used as a measure of the performance for different $M_{2}$.

As shown in Figure 3, the greater the $M_{2}$ is, the better the receiver performance is. It is because the bandwidth of the interfering signal in each frequency bin decreases with the $M_{2}$ increasing. And the greater the bandwidth of the interfering signal in each frequency bin is, the greater the probability of multiple interferences falling into one TF point is. And the number of interferences falling into one TF point exceeding the limitation that the antenna array can deal with may bring lower interference suppression performance. However, the computational complexity increases with the increase of $M_{2}$. Then, the value of $M_{2}$ should be selected by considering the computational complexity and interference suppression performance.

4.2. Advantages of the Proposed Method. In this section, the proposed multiple interference suppression method is compared with the well-known space-only MPDR (S-MPDR) beamformer [7], the distortionless space-time MPDR processor (DST-MPDR) [9], and the combining method based on double-chain quantum genetic matching pursuit-sparse decomposition and MPDR beamformer (DCQGMP-SD \& MPDR) [14]. The number of time delay taps of DSTMPDR is $N_{\tau}=9$; for the proposed method, $M_{2}=32$. And assume that we only knew the prior information of S-T and
LFMCW interfering signals, then the signal processing flow and parameters of DCQGMP-SD \& MPDR are the same as in [14].

In order to evaluate the performance of the four methods and to provide a direct and precise description, the computational complexities of each processing module of different methods are given in Table 2, where "/" represents that the module is not needed. $N_{\mathrm{J}}, N_{\mathrm{G}}$, and $N_{\mathrm{Q}}$ denote the number of interferences can be dealt with by DCQGMP-SD, the number of iterations, and the population size in the DCQGMP, respectively. $N_{s}$ and $N_{\omega}$ are the length of sampled signal data and the total number of windows occurs for the observation length $N_{\mathrm{s}}$, respectively. From Table 2, we can find that the computational complexity of the proposed method is less than that of DCQGMP-SD \& MPDR, but compared with S-MPDR and ST-MPDR, the computational complexity of the proposed method may increase. It is because the $M_{1}$ increases with the increase of $T_{c}$, the common period of multiple WBPFM interferences. Fortunately, most digital signal processors completely satisfied the required computing power for a GNSS receiver equipped with a small number of antenna elements.

To adequately show the advantages of the proposed method, four interference scenarios, denoted I, II, III, and IV, were considered. In the scenario I, interference 1,3 , and 5 are used; in the scenario II, interference $1,3,4$, and 5 are employed; in the scenario III, interference 1, 2, 


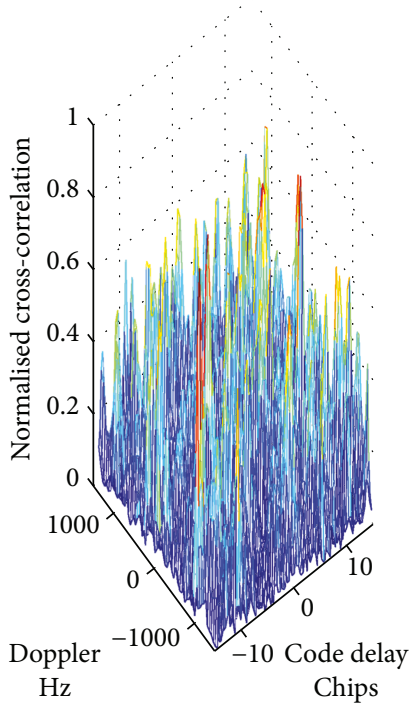

(a) S-MPDR

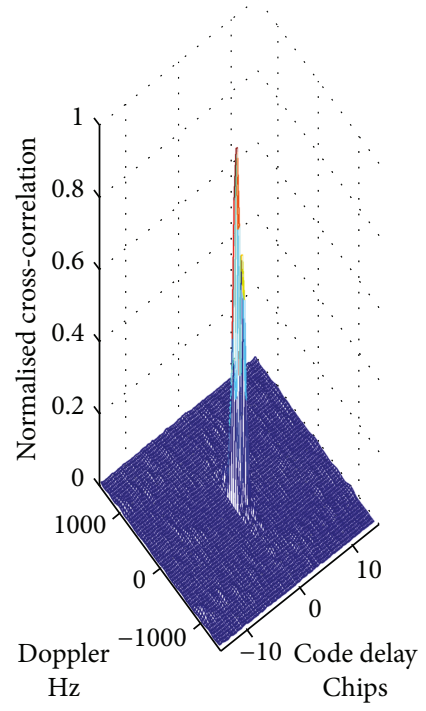

(b) DST-MPDR

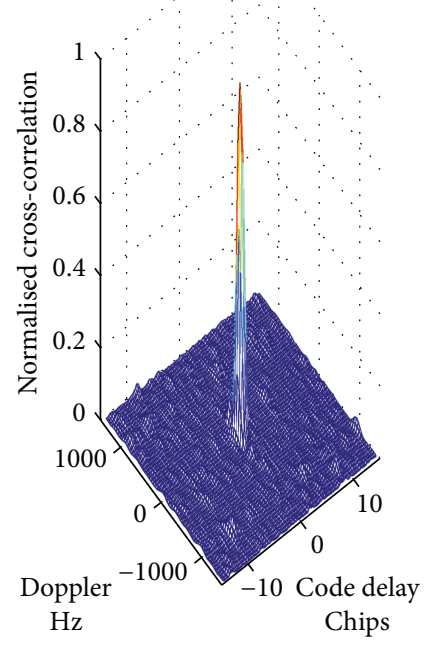

(c) DCQGMP-SD \& MPDR

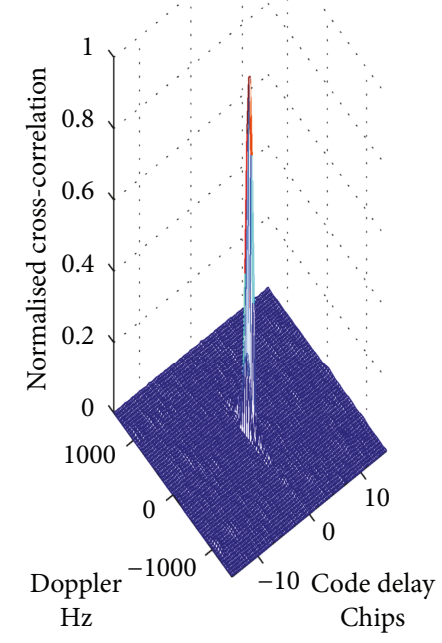

(d) Proposed

FIgURE 5: Correlation peaks after interference suppression for scenario II.

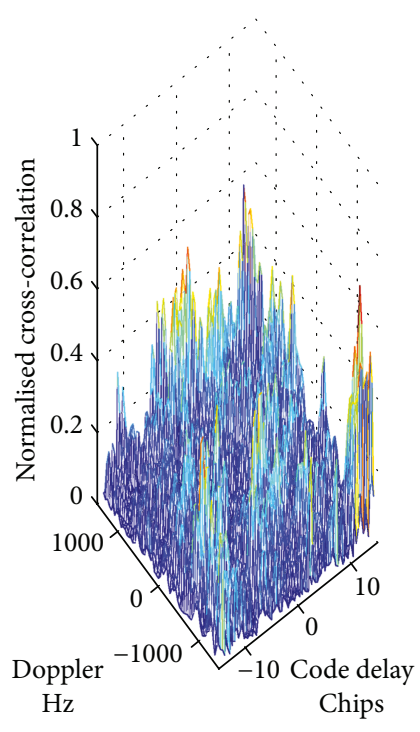

(a) S-MPDR

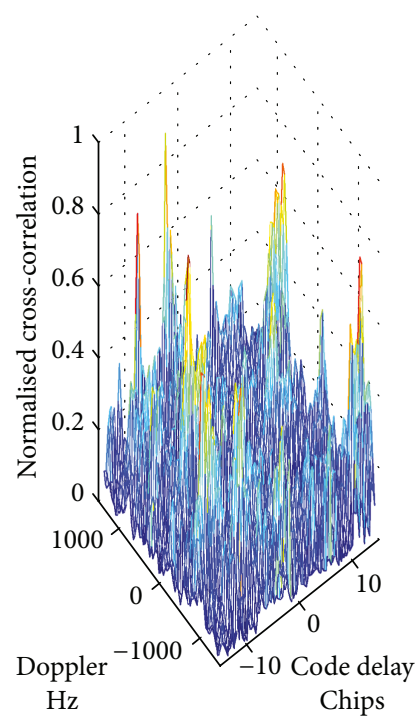

(b) DST-MPDR

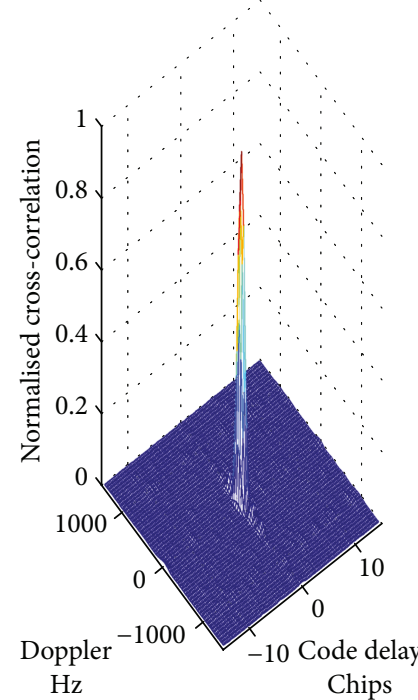

(c) DCQGMP-SD \& MPDR

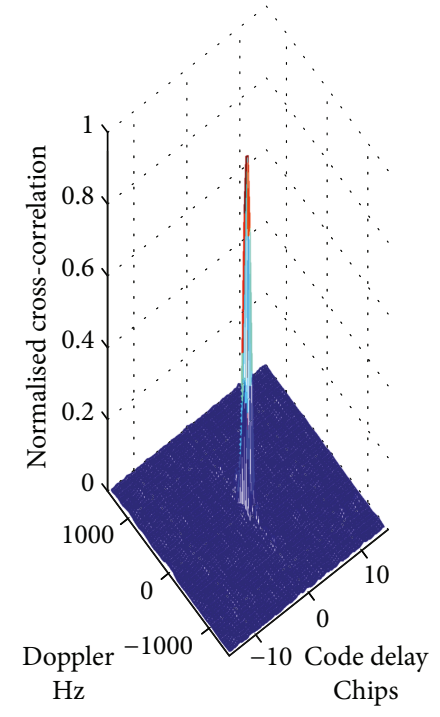

(d) Proposed

FIGURE 6: Correlation peaks after interference suppression for scenario III.

and 5 are adopted; and scenario IV consists all interferences in Table 1. And the acquisition results after interference suppression obtained by coherent integration are used to measure the performance of different methods for interference suppression. The integration time of the correlator is set to $5 \mathrm{~ms}$.

For scenario I, since the number of interferences is less than that of antenna elements, all methods are effective to suppress interferences. It is consist of the acquisition results as shown in Figure 4, where the correlation peaks of C/A code are particularly obvious.

For scenario II, although the number of interferences exceeds the DoF of the antenna array, the number of antenna array is more than that of WBI. Then, from Figure 5, it can be found out that DST-MPDR, DCQGMP-
SD \& MPDR, and the proposed method are all able to suppress these interferences, but the S-MPDR failed to deal with so many interfering signals. And for scenarios III and IV, Figures 6(a) and 6(b) and Figure 7(a) and 7(b) show that the S-MPDR and DST-MPDR failed to mitigate the interferences due to the presence of the interference with the same DOA as the GNSS signal or excessive number of WBI. From Figure 6(c), DCQGMP-SD \& MPDR can deal with the LFMCW interference with the same DOA as the GNSS signal; it is because the prior information of S-T and LFMCW interfering signals is known, and they can be canceled by sparse decomposition in the first stage of the method. But as shown in Figure $7(\mathrm{c})$, since the prior information of SFMCW interferences is unknown and the WBI is too much in the residuary interferences, after processing by DCQGMP- 


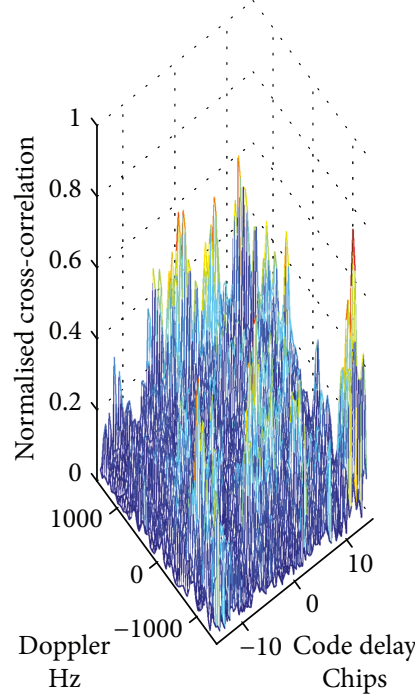

(a) S-MPDR

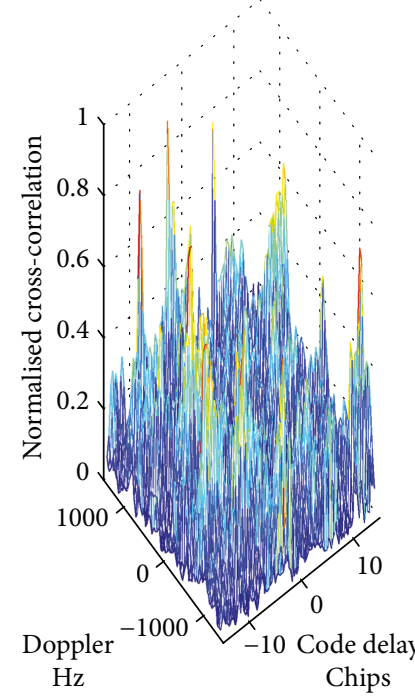

(b) DST-MPDR

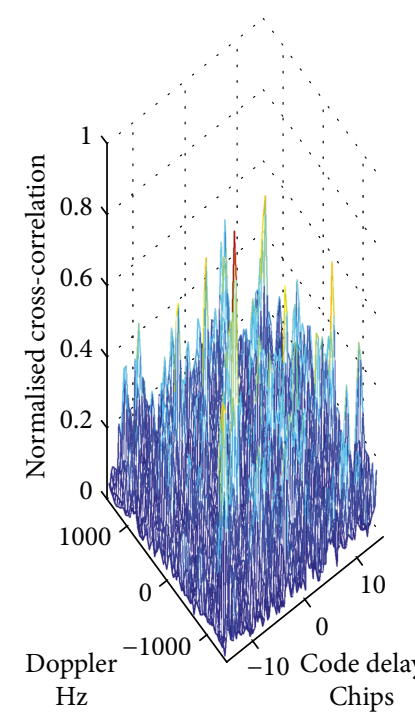

(c) DCQGMP-SD \& MPDR

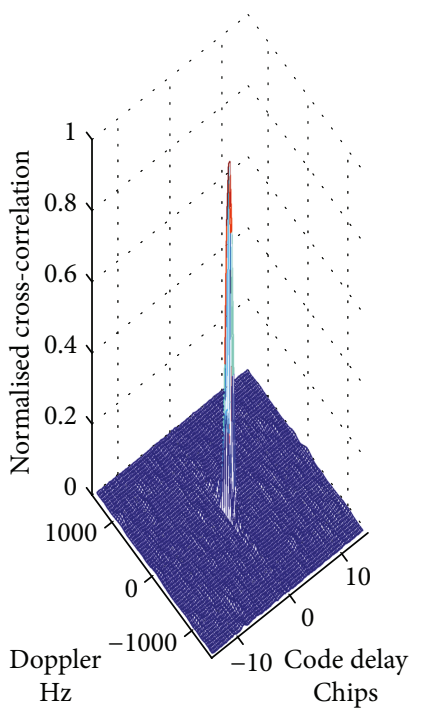

(d) Proposed

FIGURE 7: Correlation peaks after interference suppression for scenario IV.

SD, DCQGMP-SD \& MPDR failed in scenario IV. However, for both the two scenarios, from Figure 6(d) and Figure 7(d), it is obvious that the proposed STFAP can effectively deal with all interferences. It is because the idea of regrouping processing in the TF domain and the improved weight calculation technique using the information of reference TF points can make full use of the sparse characteristics of interference signals in time, frequency, and space domains.

\section{Conclusions}

Considering the different energy distribution and the periodicity of interfering signals in TF domain and their sparse property in spatial domain, a STFAP has been proposed. By regrouping the TF points according to the common period of WBPFM interferences and calculating the weight of spatial filters using the information of reference TF points, the proposed method can deal with more WBPFM interferences. Simulation results have shown that with the same configuration of an antenna array, the proposed STFAP outperforms the compared methods for multiple interference suppression. Especially, it can effectively cope with WBPFM interferences with the same DOA as the GNSS signal.

\section{Conflicts of Interest}

The authors declare that there is no conflict of interest regarding the publication of this article.

\section{Acknowledgments}

This work has been supported by the International S \& T Cooperation Program of China (ISTCP) (no. 2015DFR10220), the National Major Research \& Development project of China (no. 2016YFC0101700), the Application Technology Research and Development of Heilongjiang Science and Technology Agency (no. GX16A007), the National
Natural Science Foundation of China (no. 61371172), the Application Technology Research and Development of Heilongjiang Science and Technology Agency (no. GC13A307), and the Provincial Technique Research of Zhejiang (no. 2016C31095).

\section{References}

[1] G. X. Gao, M. Sgammini, M. Lu, and N. Kubo, "Protecting GNSS receivers from jamming and interference," Proceedings of the IEEE, vol. 104, no. 6, pp. 1327-1338, 2016.

[2] N. Varshney and R. C. Jain, "An adaptive notch filter for narrow band interference removal," in 2013 National Conference on Communications (NCC), New Delhi, India, February 2013.

[3] M. R. Mosavi and F. Shafiee, "Narrowband interference suppression for GPS navigation using neural networks," GPS Solutions, vol. 20, no. 3, pp. 341-351, 2015.

[4] Y.-R. Chien, "Design of GPS anti-jamming systems using adaptive notch filters," IEEE Systems Journal, vol. 9, no. 2, pp. 451-460, 2015.

[5] M. J. Rezaei, M. R. Mosavi, and M. Abedi, "New GPS antijamming system based on multiple short-time Fourier transform," IET Radar, Sonar \& Navigation, vol. 10, no. 4, pp. 807-815, 2016.

[6] A. Broumandan, A. Jafarnia-Jahromi, and S. Daneshmand, "Overview of spatial processing approaches for GNSS structural interference detection and mitigation," Proceedings of the IEEE, vol. 104, no. 6, pp. 1246-1257, 2016.

[7] C. Fernandez-Prades, J. Arribas, and P. Closas, "Robust GNSS receivers by array signal processing: theory and implementation," Proceedings of the IEEE, vol. 104, no. 6, pp. 1207-1220, 2016.

[8] R. L. Fante and J. J. Vaccaro, "Wideband cancellation of interference in a GPS receive array," IEEE Transactions on Aerospace and Electronic Systems, vol. 36, no. 2, pp. 549-564, 2000.

[9] S. Daneshmand, A. Jahromi, A. Broumandan, and G. Lachapelle, "GNSS space-time interference mitigation and 
attitude determination in the presence of interference signals," Sensors, vol. 15, no. 12, pp. 12180-12204, 2015.

[10] Y. Chen, D. Wang, and P. Liu, “An improved approach of SFAP algorithm for suppressing concurrent narrowband and wideband interference," in Proceedings of China Satellite Navigation Conference (CSNC) 2016 China, vol. II, pp. 6980, Springer, Singapore, 2016.

[11] J. L. Volakis, A. J. O'Brien, and C.-C. Chen, "Small and adaptive antennas and arrays for GNSS applications," Proceedings of the IEEE, vol. 104, no. 6, pp. 1221-1232, 2016.

[12] X. Wang, M. Amin, F. Ahmad, and E. Aboutanios, "Interference DOA estimation and suppression for GNSS receivers using fully augmentable arrays," IET Radar, Sonar \& Navigation, vol. 11, no. 3, pp. 474-480, 2017.

[13] Q. Guo and L. Qi, "Cascaded multitype interferences suppression method using sparse representation and array processing for GNSS receiver," International Journal of Antennas and Propagation, vol. 2017, Article ID 9832197, 17 pages, 2017.

[14] Q. Guo and L. Qi, "Combining DCQGMP-based sparse decomposition and MPDR Beamformer for multi-type interferences mitigation for GNSS receivers," Sensors, vol. 17, no. 4, p. 813, 2017.

[15] M. K. Bek, S. A. Elgamel, and E. M. Shaheen, "Analysis of the global position system acquisition process in the presence of interference," IET Radar, Sonar \& Navigation, vol. 10, no. 5, pp. 850-861, 2016.

[16] P. Wang, Y. Wang, X. Yu, and S. Wu, "Performance comparison of code discriminators in the presence of CW interference," Wireless Personal Communications, vol. 89, no. 2, pp. 405-426, 2016.

[17] H. Mao, D. W. Wei, and H. Lu, "Effectiveness analysis of the blanket jamming mode to GPS M code signal," Journal of University of Electronic Science and Technology of China, vol. 44, no. 3, pp. 350-356, 2015. 


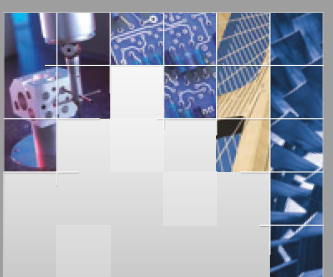

\section{Enfincering}
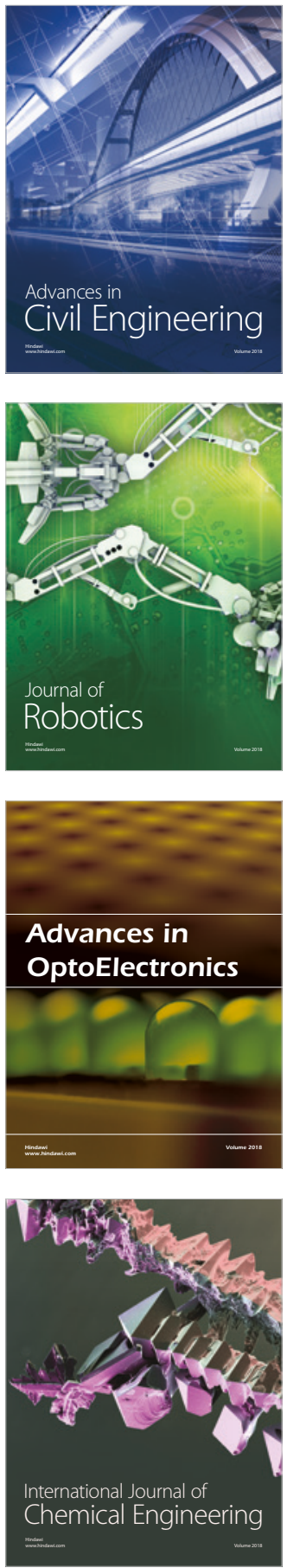

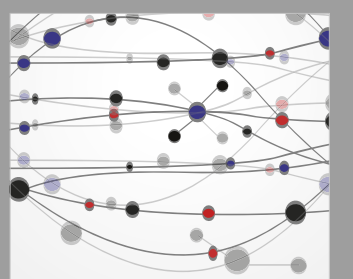

\section{Rotating \\ Machinery}

The Scientific World Journal

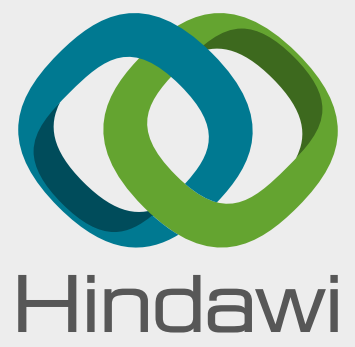

Submit your manuscripts at

www.hindawi.com
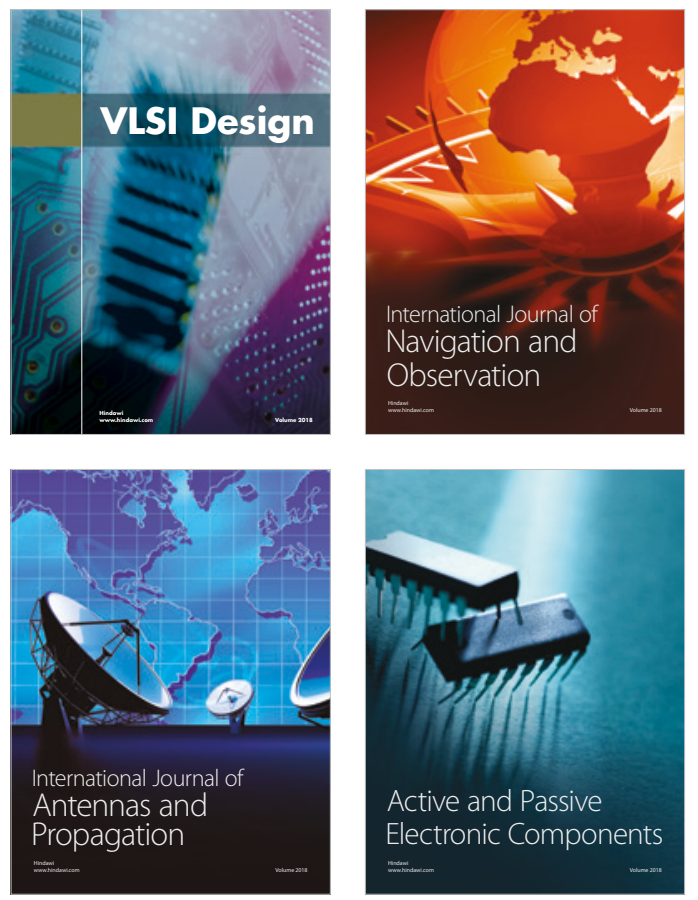
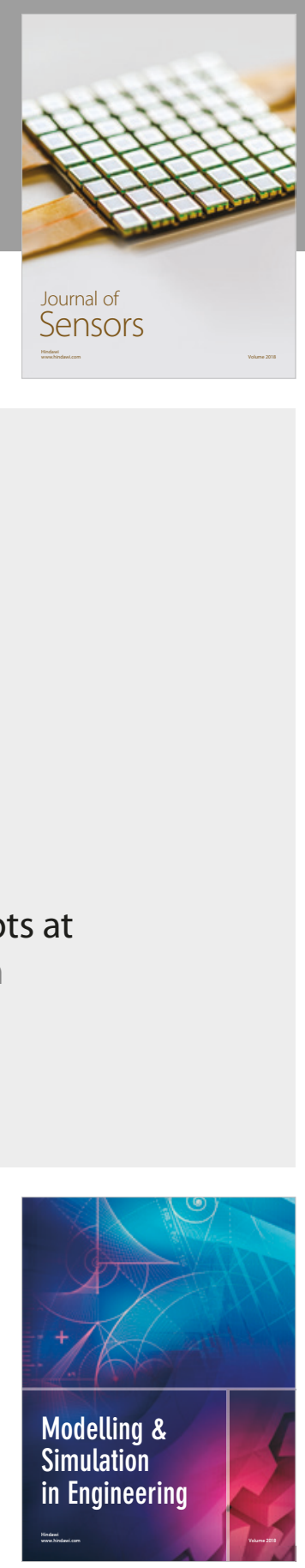

\section{Advances \\ Multimedia}
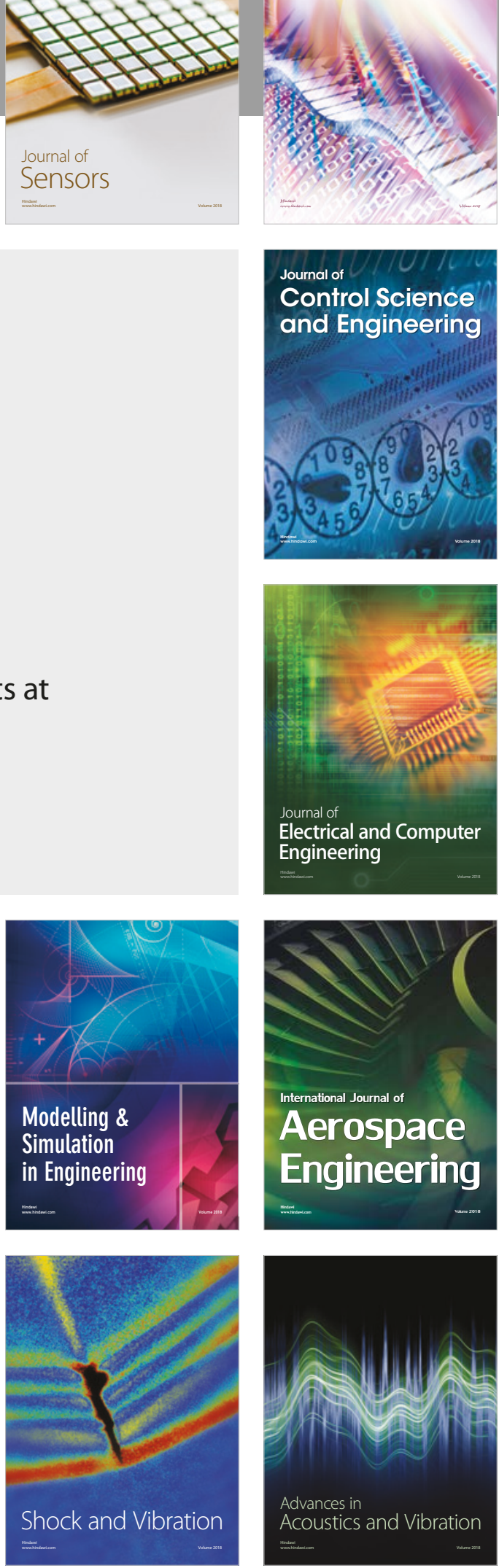